\title{
Literarische Crossings
}

\author{
Sprachliche Kreuzungen in der amerikanischen \\ Dialektliteratur des 19. Jahrhunderts
}

FLORIAN FREITAG/ANNIKA ROSBACH

\section{1. „NEGER-ENGLISCH“ FÜR DEUTSCHE LESER}

Harriet Beecher Stowes Uncle Tom's Cabin; Or, Life among the Lowly hatte bereits während seiner Veröffentlichung als Fortsetzungsroman in der amerikanischen Anti-Sklaverei-Zeitschrift The National Era von Juni 1851 bis April 1852 immensen Erfolg. ${ }^{1}$ Dies führte dazu, dass noch im Jahr der Erstveröffentlichung als Buch (1852) zahlreiche Übersetzungen in andere Sprachen angefertigt wurden, darunter Französisch, Italienisch, Spanisch, Niederländisch und Bulgarisch. Eine erste deutsche Version erschien ebenfalls bereits 1852 unter dem Titel Onkel Tom's Hütte oder Negerleben in den Sclavenstaaten des freien Nordameri$\mathrm{ka}^{2}{ }^{2}$ Wie jedoch schon der aus heutiger Sicht recht kurios anmutende Untertitel„In deutscher Auffassungsweise f[ür] deutsche Leser bearb[eitet] v[on] Dr. Ungewitter" - nahelegt, handelt es sich hierbei keineswegs um eine wortgetreue Übertragung des englischen Originaltexts ins Deutsche. So schreibt der Übersetzer, eben jener Dr. Ungewitter, in seinem Vorwort:

1 Vor allem dank Stowes Roman gelang es der wöchentlich erscheinenden Zeitschrift, ihre Auflage von 15.000 Exemplaren (1850) auf 19.000 Exemplare (1852) zu steigern. Vgl. HARROLD, 2011, S. 67. Zur Publikationsgeschichte des Romans allgemein vgl. PARFAIT, 2007.

2 Zur Rezeption von Uncle Tom 's Cabin in Deutschland vgl. PAUL, 2005, S. 127-185. 
Das englische Original [...] ist 329 enggedruckte Octavseiten stark; und eine getreue und vollständige Uebersetzung desselben würde dem deutschen Leser schwerlich sehr behagen; schon wegen der vielen und langen, im Provinzialdialekt und Neger-Englisch geführten Gespräche, die freilich dem nordamerikanischen und englischen Leser sehr zusagen mögen, weil er nordamerikanisch oder englisch denkt und fühlt $[\ldots]$, die aber für den deutschen Leser ermüdend sind, indem sie ihn in einen Ideenkreis führen, der ihn durchaus nicht interessieren kann. Sie würden, vollständig wiedergegeben, auf ihn ungefähr den nämlichen Eindruck machen, wie auf den Engländer oder Nordamerikaner plattdeutsche Gespräche zwischen Bauern in den deutschen Marschländern der Nordseeküste bei $\mathrm{Ab}$ schließung eines Mastochsen- oder Pferdehandels. [...] Er [der deutsche Leser] erhält demnach das englische Original in einer abgekürzten deutschen Bearbeitung, in der jedoch nichts Wesentliches ausgelassen ist, und die dem deutschen Leser im deutschen Sinne und in deutscher Auffassungsweise genau wiedergibt, was das Original mit vielem Wortschwall und häufigen Abschweifungen erzählt. ${ }^{3}$

Während Ungewitters deutsche „Bearbeitung“ von Stowes Roman in manchen Gesichtspunkten auch überaus pedantisch erscheinen mag (so übersetzt er den Vornamen der Autorin von „Harriet“ in „Henriette“), verfährt sie in anderen Fällen geradezu sorglos und unbekümmert. Letzteres trifft insbesondere auf die "Wortschwälle" zu, die zahlreichen Dialogpassagen in Uncle Tom's Cabin, die nicht bzw. nur teilweise im Standardenglischen verfasst sind. Sie werden vom Übersetzer entweder im Standarddeutschen wiedergegeben oder schlichtweg durch kurze Zusammenfassungen des Erzählers (ebenfalls im Standarddeutschen) ersetzt. Dabei ist durchaus fraglich, ob Ungewitter tatsächlich die von Stowe im „Provinzialdialekt“ und im „Neger-Englisch“ verfassten Gespräche als für deutsche Leser ermüdend und uninteressant erachtete oder ob er sich nicht vielmehr außer Stande sah, das nicht-standardsprachliche Englisch in einen deutschen Kontext zu übertragen und seine Lösung dieses translatorischen Problems im Nachhinein zu rechtfertigen suchte.

Jene „Wortschwälle“ in nicht-standardsprachlichen Varietäten des Englischen, an denen sich Ungewitter in Uncle Tom's Cabin so störte, sollten jedoch im weiteren Verlauf des 19. Jahrhunderts eine immer bedeutendere Rolle in der amerikanischen Literatur spielen: „Late-nineteenth-century America was crazy about dialect literature, "4 konstatiert der amerikanische Philologe Gavin Jones in seinem Buch Strange Talk. The Politics of Dialect Literature in Gilded Age America. Die Verwendung von Lekten jedweder Art - Jones verwendet „Dia-

4 JONES, 1999, S. 1. 
lekt" als eine Art Oberbegriff für Regio-, Sozio-, Ethno- und Chronolekte ${ }^{5}$ - beschränkte sich dabei keineswegs auf Romane bzw. Prosa; auch in Kurzgeschichten, in der Lyrik und auf der Bühne erfreuten sich nicht-standardsprachliche Varietäten und insbesondere Dialekte zunehmender Beliebtheit.

Neben Stowes „Provinzialdialekt“ (womit Ungewitter vermutlich die in den Südstaaten der USA gesprochene Sprachvarietät meinte) und ihrem „NegerEnglisch" fanden u.a. auch die regionalen Varietäten des Englischen in Kalifornien (z.B. in den Kurzgeschichten von Bret Harte), in Neuengland (in den Romanen und Kurzgeschichten von Sarah Orne Jewett) und im Mittelwesten (bei Hamlin Garland) Eingang in die Literatur. Hinzu kamen die von den eigentlich frankophonen Kreolen und Cajuns in Louisiana gesprochenen Varietäten des Englischen (etwa in den Werken von Kate Chopin, Grace E. King oder George Washington Cable). Ähnlich wie im Fall von Uncle Tom's Cabin waren Diaund andere Lekte vor allem auf die direkte Rede von Charakteren beschränkt. Da jedoch manche Werke wie z.B. Joel Chandler Harris' „Uncle Remus“-Geschichten (ab 1880) oder Thomas Nelson Pages Gedicht „Uncle Gabe's White Folks“ (1877) (nahezu) ausschließlich aus direkter Rede bestehen, verschwindet hier die Standardsprache völlig.

Nichtsdestotrotz spielte selbst in den zuletzt genannten Texten das Standardenglische als Referenzpunkt eine wichtige Rolle. Ausgehend von den dazugehörigen Orthographie- und Aussprachekonventionen versuchten die Verfasser von Dialektliteratur nämlich, nicht-standardsprachliche Varietäten lautmalerisch und durch kreative Abwandlungen der Rechtschreibung darzustellen. ${ }^{6}$ Sie bauten somit auf die im 19. Jahrhundert nicht unübliche Praxis, Texte laut zu lesen. Wenn sie die Texte (zumindest in Gedanken) laut vorlasen und dabei den Aussprachekonventionen der Standardsprache folgten, so konnten die Leser, so das implizite Versprechen der Texte, den Klang der jeweils dargestellten Varietät zumindest erahnen.

Bei der Lektüre von Dialektliteratur wurden Leser im 19. Jahrhundert - zumindest jene, die den im Text transkribierten Lekt sonst nicht verwendeten somit zu einer Art Sprachkreuzung gezwungen. ${ }^{7}$ In seinem Buch Crossing. Language and Ethnicity among Adolescents definiert der britische Linguist Ben Rampton language crossing als die Verwendung von Sprachen oder Sprachvari-

5 EBD., S. 12.

6 Vgl. ROSENWALD, 2008, S. 9f.

7 Fur das von Rampton beschriebene language crossing verwenden wir nach Hinnenkamp die Lehnübersetzungen „Sprachkreuzung“ bzw. „sprachliche Kreuzung“. Vgl. HINNENKAMP, 1998, S. 150-153. 
etäten, die mit bestimmten regionalen, sozialen und/oder ethnischen Gruppen assoziiert werden, denen der Sprecher - bzw. hier der Leser - weder angehört noch als zugehörig empfunden wird. ${ }^{8}$ Für Leser, denen es dazu gelang, den für sie fremden Lekt gedanklich in die eigene Sprache zu übersetzen und damit die encodierten Inhalte zu verstehen, dürfte die Lesefreude ungleich höher gewesen sein als für Leser, die an der Entzifferung des Lekts scheiterten und sich deshalb aus der fiktiven Welt ausgeschlossen gefühlt haben dürften.

$\mathrm{Ob}$ die Leser jedoch tatsächlich den Klang des betreffenden Lektes auf der Grundlage der Transkriptionen zumindest annähernd reproduzieren konnten, ist äußerst fraglich. Denn zum einen geht dem Crossing bei der Lektüre literarischer Lekte zumeist auch ein Crossing des Autors voraus. Die Mehrzahl der Verfasser von amerikanischer Dialektliteratur des 19. Jahrhunderts hatte nämlich zwar persönlichen Kontakt mit Sprechern der jeweiligen Varietät, gehörte jedoch selbst nicht dieser Sprechergruppe an. So imitierte beispielsweise Stowe als weiße, in Connecticut im Nordosten der heutigen Vereinigten Staaten geborene Schriftstellerin das Southern White English weißer Plantagenbesitzer ebenso wie das von den Sklaven in den südlichen Staaten gesprochene Black English. Und George Washington Cable, der zwar in New Orleans geboren wurde und aufwuchs, jedoch anglo-amerikanischer Abstammung war, stellte in seinen Werken Black English, das Englische der Kreolen und Cajuns sowie das Kreolfranzösische und viele Zwischenformen dar. Zum anderen hing die Qualität der Transkriptionen auch mit deren Funktion in den Texten zusammen, die ihnen die Autoren (bewusst oder unbewusst) zuschrieben. Während sich etwa Cable um eine akribische Nachahmung möglichst vieler lexikalischer, phonologischer und grammatikalischer Lektspezifika bemühte, griff Stowe gezielt auf Einzelmerkmale zurück, die für den Leser von hohem Widererkennungswert waren, weil sie stereotyp mit den Sprechern der Varietät assoziiert wurden.

In diesem Kontext möchten wir uns im Folgenden zunächst allgemein mit der Dialektliteratur als Form der sprachlichen Kreuzung auseinandersetzen und wesentliche Gemeinsamkeiten und Unterschiede zu Ramptons ursprünglichem Konzept und dessen Rezeption durch andere Forscher beleuchten. Danach soll insbesondere unter Rückgriff auf die Arbeiten von Gavin Jones und Brian Hochman zur amerikanischen Dialektliteratur des späten 19. Jahrhunderts die Multifunktionalität von literarischen Lekten herausgearbeitet werden. Hier wird sich zeigen, dass, wie Jones schreibt, ,[d]ialect satisfied a vast range of motivations “. ${ }^{9}$ Im Anschluss diskutieren wir zwei Fallbeispiele: Harriet Beecher Stowes

8 RAMPTON, 1995, S. 14; DERS., 1998, S. 291.

9 JONES, 1999 , S. 7. 
Uncle Tom's Cabin (1852) sowie George Washington Cables The Grandissimes (1880) - zwei Dialektromane also, die beide in den Südstaaten der USA spielen und jeweils mehrere der dort gesprochenen Varietäten des Englischen darstellen. Obwohl sowohl Stowe als auch Cable in ihren Romanen Lekte gezielt einsetzen, um gegen die Sklaverei (Stowe) und für die Anerkennung der Rechte von Afroamerikanern im Süden in der Zeit nach dem Bürgerkrieg (Cable) zu schreiben, so tun sie dies, wie wir zeigen werden, auf höchst unterschiedliche Weise. Abschließend werden wir darauf eingehen, welche weiteren Sprachkreuzungen die Crossings in Stowes und Cables Romanen zeitigten.

\section{LITERARISCHES CROSSING}

Eine Analyse von Dialektliteratur als Sprachkreuzung setzt eine signifikante Modifikation bzw. Ausweitung von Ramptons Crossing-Konzept voraus. Die Fälle von Sprachkreuzungen, die Rampton in seinem Buch untersucht, sind ausschließlich dem Bereich der gesprochenen Sprache zuzuordnen; Androutsopoulos spricht folgerichtig vom „konversationellen Gebrauch von einer Sprache oder Sprachvarietät, die dem Sprecher ethnisch bzw. sozial nicht eigen ist““. ${ }^{10}$ Nun kann man festhalten, dass nicht-standardsprachliches Englisch in der amerikanischen Dialektliteratur ausschließlich in der direkten Rede einzelner Charaktere verwendet wird. Dennoch stellt die Verwendung natürlich eine verschriftlichte Form des Crossings dar, noch dazu im ästhetischen Kontext der Literatur (statt in Alltagsgesprächen), weshalb wir hier von literarischem Crossing sprechen.

Hieraus ergibt sich ein weiterer Unterschied zu Ramptons Begriff der sprachlichen Kreuzung. Anders als beim mündlichen Crossing ist der Gebrauch der nicht-standardsprachlichen Varietät bei der literarischen Sprachkreuzung etwa nicht nur auf einzelne sprachliche Einsprengsel oder "Sprachfetzen“ - in Ramptons Beispielen oftmals nur einzelne Worte - beschränkt. Stattdessen werden Standardsprache und nicht-standardsprachliche Varietäten klar bestimmten Rollen im Text zugeordnet, z.B. dem Erzähler und individuellen Charakteren, die jeweils ausschließlich die entsprechende Sprache bzw. Sprachvarietät in vollem Umfang verwenden. Während „mündliche" Kreuzungen, wie Androutsopoulos feststellt, somit ,mit einer minimalen Kompetenz auskommen, die vor allem Routineformeln und Wortschatzelemente, aber auch lautliche und prosodische Merkmale umfasst ${ }^{6}{ }^{11}$ mussten Verfasser von Dialektliteratur daher die einschlä-

10 EBD., S. 9; Hervorhebung der Autoren.

11 EBD., S. 30. 
gige Sprache weitaus umfassender beherrschen (oder zumindest diesen Anschein erwecken).

Andererseits lassen sich jedoch auch Parallelen zwischen Ramptons ,klassischem" Konzept des Crossing und dem literarischen Crossing ausmachen. So geht etwa Androutsopoulos im ersten Falle generell von einem ,ambivalenten Verhältnis von Sprecher und Code bzw. Stimme“ aus. ${ }^{12}$ Häufig seien Sprecher und Stimme hier klar voneinander getrennt, Androutsopoulos spricht gar von einer „Dissoziation von Sprecher und Stimme“. Dies bedeute jedoch nicht, dass der Sprecher die fremde Stimme nicht auch als Teil der eigenen Identität annehmen könne. ${ }^{13}$ Bei der literarischen Sprachkreuzung lässt sich eine ähnliche Ambivalenz ausmachen. So argumentiert der Literaturwissenschaftler Bill Hardwig am Beispiel der amerikanischen Autoren Mary Noailles Murfree und Charles Chesnutt, dass die Lektüre von Dialektliteratur ihren Reiz zumindest zum Teil aus der implizierten Annahme zog, ,that one was reading the work of a writer who has emerged from the culture about which s/he wrote" - eine Annahme, der etwa Murfree, die über das raue Leben in der Appalachenregion schrieb, durch die Wahl eines entsprechenden Pseudonyms Rechnung trug. ${ }^{14}$ Tatsächlich jedoch handelte es sich bei den Verfassern in den wenigsten Fällen um der jeweiligen Sprechergruppe Zugehörige, sondern bestenfalls um kenntnisreiche oder interessierte „Experten“. Einen interessanten Fall stellt Hamlin Garland dar, der zwar auf einer Farm im mittleren Westen der USA aufwuchs, in seiner berühmten Kurzgeschichte „Up the Coulee. A Story of Wisconsin“ (1891) jedoch mit der Figur des Rückkehrers die allmähliche Entfremdung von den eigenen regionalen kulturellen Wurzeln (und damit auch von der regionalen Sprache) literarisch verarbeitete. ${ }^{15}$

Ferner stellt Rampton fest, dass Sprachkreuzungen an bestimmte Erscheinungskontexte gebunden sind: ,language crossing was located in moments when the ordered flow of social life was loosened and normal social relations could not be taken for granted. ${ }^{16}$ Androutsopoulos erläutert, dass es sich hierbei insbesondere um liminale Momente wie „Übergangsphasen, in denen die Rollen und Aufgaben der Beteiligten nicht klar definiert sind, Spiele und künstlerische Performance, rituelle Beschimpfungen, der Bruch normativer Erwartungen und der

12 EBD., S. 23.

13 EBD. S. $23 \mathrm{f}$.

14 HARDIWIG, 2013, S. 45.

15 Vgl. Garland, 1918, S. 67-129.

16 RAMPTON, 1995, S. 193. 
Ausdruck starker Gefühle" handelt. ${ }^{17}$ Nun lassen sich das Verfassen und die Lektüre (insbesondere das laute Vorlesen) von Dialektliteratur durchaus als „künstlerische Performance[s]“ auffassen. Wichtiger und aufschlussreicher erscheint jedoch ein anderer Kontext: Die zweite Hälfte des 19. Jahrhunderts - der Zeitraum also, in dem die Dialektliteratur besondere Erfolge feierte - stellte in den USA eine Epoche weitreichender wirtschaftlicher und sozialer Umbrüche dar. Die Abschaffung der Sklaverei nach dem Bürgerkrieg, zunehmende Industrialisierung und Urbanisierung sowie die massive Einwanderung insbesondere aus Europa stellten die amerikanische Gesellschaft vor enorme Herausforderungen, im Zuge derer auch die Verhältnisse zwischen verschiedenen sozialen Gruppen, u.a. zwischen Schwarz und Weiß, Einheimischen und Immigranten, ländlicher und urbaner Bevölkerung, neu austariert werden mussten. Wie die Forschung zur amerikanischen Dialekt- und local color-Literatur seit den frühen 1990er Jahren in mehreren Schritten herausgearbeitet hat, sind es exakt diese Konflikte, die in den Texten mittels der Darstellung von interkulturellen Begegnungen zwischen „regionalen" und „kosmopolitischen" Charakteren aufi imaginativer Ebene ausgehandelt werden. ${ }^{18}$ Ähnlich wie das mündliche Crossing auf der Mikroebene des Gesprächs zwischen Einzelpersonen lässt sich somit auch die literarische Sprachkreuzung, hier jedoch auf der gesamtgesellschaftlichen Makroebene, mit liminalen Situationen des Übergangs und der Neuverteilung von sozialen Rollen verbinden.

Die weitaus wichtigste Parallele zwischen „klassischem" und literarischem Crossing stellt jedoch deren Multifunktionalität dar. So schreibt Androutsopoulos mit Bezug auf „,klassisches“ Crossing:

Im Hinblick auf die Beweggründe für Kreuzungen deutet die Diskussion auf ein komplexes Motivationsgeflecht hin. [...] Kreuzungen können je nach Konstellation als integrierend oder ausgrenzend, ,emanzipatorisch“" oder ,reaktionär" erscheinen. ${ }^{19}$

Ein ähnlich vielschichtiges „Motivationsgeflecht“ bzw. eine ähnliche Multifunktionalität lassen sich auch bei der Verwendung nicht-standardsprachlicher Varie-

17 ANDROUTSOPOULOS, 2002, S. 9. Androutsopoulos merkt jedoch auch an, dass „Liminalităt mehr als empirisch nützliche Generalisierung für präferierte Erscheinungskontexte und weniger als ein Definitionsmerkmal für Crossing" verstanden werden sollte (EBD., S. 10).

18 Vgl. FREITAG, 2013, S. 409-412.

19 ANDROUTSOPOULOS, 2002, S. 31. 
täten in der Dialektliteratur beobachten, wie im Folgenden unter Rückgriff auf die Arbeiten von Jones und Hochman gezeigt werden soll.

\section{ZUR FUNKTIONALISIERUNG VON LEKTEN IN DER DIALEKTLITERATUR}

Die Grundfunktion von Dialekten in fiktionalen Texten besteht in einer „Simulation nähesprachlicher Kommunikation “20. Die dazugehörigen Funktionen literarischer Dialektverwendung reichen sodann vom rein mimetischen Anspruch authentischer Sprachwiedergabe im Dienste eines gesteigerten Realismus-Effekts über den Einsatz als ästhetisches Element zur Verlebendigung des literarischen Stils bis hin zur symbolträchtigen Bedeutungsträgerschaft für den Text als werkimmanent. Sie können sich auch auf seinen Entstehungskontext als kommentatorisch und/oder auf die Rezeption und Wirkung des Textes durch bzw. auf den Leser als rezeptionsästhetisch beziehen. Werkimmanent dienen Dialekte in der Regel der Charakterisierung und Kontrastierung von Figuren; vor allem insofern diese sich durch den Grad ihrer Dialektintensität zu Sprachgemeinschaften bekennen oder sich von diesen abgrenzen bzw. abzugrenzen versuchen. In kommentatorischer Funktion können Dialekte Perspektiven (des Autors/der Figuren) auf sprachliche Konventionen, Normen und Hierarchien - und damit freilich auf die Kultur, deren Teil die Sprache ist - zum Ausdruck bringen. Rezeptionsästhetisch erzeugen Dialekte je nach ihrem Wiedererkennungswert für den Leser emotionale Nähe oder Distanz zum bzw. vom Text.

Diese Funktionen literarischer Dialekte lassen sich in Texten der amerikanischen Dialektliteratur aus der zweiten Hälfte des 19. Jahrhunderts schwerlich voneinander trennen. Dennoch stellen Brian Hochman ${ }^{21}$ und Gavin Jones ${ }^{22}$ vor dem Hintergrund der bereits angesprochenen politischen und gesellschaftlichen Ereignisse und Themen des Gilded Age - Rassenpolitik, Westexpansion, fortschreitende Industrialisierung, Zuwanderung aus Europa - zwei unterschiedliche Tendenzen fest; zum einen ein gesteigertes Interesse an einer möglichst realitätsgetreuen schriftlichen Aufzeichnung von Dialekten (Hochman), zum anderen einen kreativen Umgang bzw. die spielerische Abwandlung von Dialekten aus politischen, moralischen und nationalistisch-ideologischen Motivationen (Jones).

20 FREUNEK, 2007, S. 116.

21 HOCHMAN, 2010.

22 JONES, 1999. 
Hochman etwa liest George Washington Cables The Grandissimes im Kontext zeitgenössischer Versuche, Sprachvarietäten und Gesänge mittels audiotechnologischer Erfindungen - allen voran Edisons 1877 entwickeltem Phonographen - phonetisch exakt zu erfassen. The Grandissimes, so Hochman, sei als experimentelle Antwort Cables auf die Frage nach den Grenzen der auditiven Wahrnehmung und der schriftlichen Erfassbarkeit gesprochener Sprache durch Nichtmuttersprachler zu lesen. Damit habe Cable aktuelle wissenschaftliche Diskussionen zur interkulturellen „Taubheit“ verarbeitet, d.h. der Unfähigkeit, sprachliche Phänomene über die Grenzen des eigenen Sprachsystems hinaus zu verstehen, woraus nicht nur Verständigungs-, sondern auch interkulturelle Verstehensprobleme resultierten. ${ }^{23}$ Die Grenzen einer vollkommen realistischen Reproduzierbarkeit von Dialekten in literarischen Texten, so Hochman, waren für Cable somit schon dadurch gegeben, dass er als Autor niemals alle Merkmale eines von ihm nicht muttersprachlich beherrschten Dialektes würde darstellen können, weil er nicht alle von ihnen hören konnte.

Während die Auswahl bestimmter Dialektmerkmale in The Grandissimes für Cable somit ein quasi unvermeidbarer Vorgang war, selektierten und beschränkten sich andere amerikanische Autoren in der zweiten Hälfte des 19. Jahrhunderts bewusst auf Dialektcharakteristika mit hohem Wiedererkennungswert für den Leser. Dies mag einerseits den Einfluss des Realismus in der amerikanischen Literatur des 19. Jahrhunderts und das für diese Strömung charakteristische Bemühen um die Glaubwürdigkeit des Fiktionalen widerspiegeln. Denn durch die Schaffung einer Illusion linguistischer Authentizität werden die Leser eher geneigt sein, zu glauben, das Erzählte habe sich genau so in einer bestimmten Region oder einem bestimmten Milieu zu(ge)tragen (können). Und die Leser nehmen diese Illusion umso bereitwilliger an, je eher sich die Darstellung mit ihren Erwartungen deckt - so lässt sich hier von einer, wahrgenommenen Authentizität' sprechen. Andererseits eröffneten sich durch selektive Merkmalsetzung und deren kreative Abwandlungen, insbesondere in Form sprachlicher Kreuzungen ebenso wie in realsprachlichen Kontexten, Möglichkeiten der ,purposeful symbolic evocation" ${ }^{24}$ d.h. einer über dialektale Nuancierungen aufgespannten symbolischen Dimension. Genau diese bewusste Merkmalsetzung beschreibt Gavin Jones als herausragendes Merkmal der US-amerikanischen Dialektliteratur nach dem Bürgerkrieg.

Aus einem sprachpolitischem Betrachtungswinkel ordnet Jones den literarischen Vernakularkult nach dem Bürgerkrieg in die politische, wissenschaftliche 
und öffentliche Debatte über die Art und den Status der in den Vereinigten Staaten gesprochenen Dialekte vs. einer mittels Standardisierungsversuche angestrebten amerikanischen Nationalsprache ein. „Celebrations of the democratic realism and rugged raciness of subaltern speech met anxieties that dialect signaled the cultural fragmentation and deterioration of the nation. ${ }^{625}$ Vor jenem Hintergrund der Zelebrierung dialektaler Diversität einerseits und der Angst vor linguistischer Fragmentierung andererseits seien literarisch verwendete Dialekte das Medium für politische, moralische und ideologische Positionierungen gewesen: Autoren schrieben ,for the sake of dialect“. ${ }^{26}$

Das gesteigerte Interesse weißer Autoren an der Darstellung des Afroamerikanischen in den 1880er Jahren sei zudem Ausdruck der Auseinandersetzung mit rassenbasierten Sprachtheorien gewesen, welche die Sprachen und damit die ,Rassen“, denen sie zugeordnet wurden, gemäß ihrer ,Entwickelt- und Zivilisiertheit" hierarchisch anzuordnen versuchten. So schufen sie ein ,,racially based manifest linguistic destiny“ ${ }^{27}$ Dabei dienten vor allem ethnische Stereotypen als Ausgangspunkt für die literarische Darstellung ethnischer Lekte. Dass gerade die Sprache der ehemaligen schwarzen Sklaven ganz unten in der sprachlichen und gesellschaftlichen Hierarchie anzuordnen war, versuchten weiße Autoren der in den Südstaaten populären Plantagenliteratur durch eine Überzeichnung des Black English zu belegen. ${ }^{28}$ Der Lekt schwarzer Figuren ist in diesen Romanen oftmals nur schwer entzifferbar und durch extreme Merkmalsdichte, fehlerhafte Formen des Standardenglischen und Vulgarismen gekennzeichnet. Durch die bewusste Fehlrepräsentation und damit die Exotisierung der von der weißen Gesellschaft ohnehin als fremdartig empfundenen afroamerikanischen Sprache wurden nicht nur gesellschaftlich verankerte rassistische Stereotypen bezüglich der angeblichen Naivität, Dummheit, Clown- und Tölpelhaftigkeit der Schwarzen bestätigt, sondern auch die gesellschaftliche Nichtintegrierbarkeit jener ehemaligen Sklaven propagiert.

Im Gegensatz dazu schuf Stowe mit Uncle Tom's Cabin bereits Jahrzehnte vor dem von Jones betrachteten Zeitraum ein Beispiel par excellence für die hochpolitische Verwendung regional und ethnisch markierter Lekte. Durch multiple, den gängigen ethnischen Stereotypen ihrer Zeit zuwiderlaufende sprachli-

25 JONES, 1999, S. 209.

26 EBD., S. 2.

27 EBD., S. 23.

28 Vgl. z.B. THOMas Nelson Page, In Ole Virginia (1887) sowie Thomas Dixon mit seiner Romantrilogie The Leopard's Spots (1902), The Clansman (1905) und The Traitor (1907). 
che Kreuzungen auf Figurenebene begründet der Roman eine Form des Antirassismus, wie ihn Rampton auch für realweltliche Kreuzungssituationen festgestellt hat. ${ }^{29}$

\section{Weisse Sprache - schwarze Sprache? SPRACHKREUZUNGEN IN UNCLE TOM'S CABIN}

Dass viele der Dialoge in Harriet Beecher Stowes 1852 erschienenem Roman Uncle Tom's Cabin das Produkt sprachlicher Kreuzungen der Autorin sind, merkt man dem Text an. So werden verschiedenste Merkmale sowohl des Black English als auch der weißen Südstaatenvarietät nicht konsistent verwendet; und das obwohl Stowe für die Aufbereitung der The National Era-Version für die Buchpublikation zahlreiche Änderungen vornahm, um eben jene Merkmalsvariationen zu reduzieren. ${ }^{30}$ Besonders schwer getan zu haben scheint sich Stowe in puncto Konsistenz mit dem afroamerikanischen Ethnolekt. Sprachliche Inkonsistenzen fallen in der Sprache einzelner Figuren auf, z.B. wird das standardamerikanische Pronomen you alternierend mit seiner Lektform ye verwendet. Vor allem aber sind sie beobachtbar im Vergleich der Sprache verschiedener schwarzer Charaktere: Uncle Toms direkte Rede ist stärker lektifiziert als die der Mulattenund Quadronenfiguren, ${ }^{31}$ aber weniger merkmalsgeprägt als beispielsweise die der Aufsehersklaven auf der Plantage von Simon Legree. Im recht übersichtlichen Korpus akademischer Beschäftigung mit der Sprache der Dialoge in Uncle Tom's Cabin wird ausschließlich auf diese Ungenauigkeiten fokussiert und der Autorin eine ,notably faulty“ ${ }^{32}$,"crude ${ }^{633}$ und im Fazit unrealistische Verwendung des Black English bescheinigt. Die Mängel hinsichtlich Realismus und Konsistenz werden dabei mal mit Stowes fehlendem sprachlichen Insiderwis$\operatorname{sen}^{34}$ - Stowe stammte aus den Nordstaaten, lebte aber fast zwei Jahrzehnte an der Grenze zum Sklavenstaat Kentucky -, mal mit einem angeblichen Desinte-

29 RAMPTON, 1995, S. 21.

30 Vgl. KIRKHAM, 1977, S. 161 und S. 233-244.

31 Die Begriffe Mulatte/Mulattin und Quadrone/Quadronin gelten heute als diskriminierend, waren aber zur Entstehungszeit von Uncle Tom's Cabin und bis ins 20. Jahrhundert gebräuchliche Bezeichnungen für Sklaven mit einem schwarzen und einem weiBen Elternteil bzw. mit zu einem Viertel afrikanischer Herkunft.

32 MCDOWELL, 1931, S. 325.

33 FLOREY, 1986, S. 20.

34 MCDOWELL, 1931, S. 325; FLOREY, 1986, S. 21. 
resse der Autorin an einer präzisen Darstellung begründet. ${ }^{35}$ Der Vorwurf, Stowe hätte fernab jedweder Realität und unsauber gearbeitet, ist inzwischen in verschiedenen Publikationen widerlegt worden ${ }^{36}$ Hartnäckig hält sich in der UncleTom-Forschung jedoch die Ansicht, dass die Sklavenfiguren im Roman u.a. aufgrund ihrer Sprache zum Stereotyp des lüsternen, clownhaften, kindlich-naiven und vor allem dummen Schwarzen degradiert worden seien. ${ }^{37}$

Allen Studien zur Authentizität und Stereotypisierung der Sprachgebung in Uncle Tom's Cabin ist gemein, dass sie Stowes Roman als realistischen Text lesen - und damit genau so, wie er inhaltlich nicht zu verstehen ist. Zwar verorten realistische Details wie Haus- und Landschaftsbeschreibungen und eben auch die Verwendung realweltlicher Lekte das Romangeschehen im Amerika der 1850er Jahre. Doch die Geschichte von Uncle Tom transzendiert die Realität insofern, als sie eine Analogie zwischen der Sklaverei in Amerika und dem Sündenfall der Menschheit zieht. ${ }^{38}$ In diesem allegorischen Narrativ kommt den Lekten eine Schlüsselfunktion zu: Die bislang als Inkonsistenzen kritisierte Variation in der Intensität der Lektfärbung ist eine bewusste Nunancierung, die auf einem an moralischen Werten orientierten anti-stereotypen Sprachgebungskonzept basiert, das den Zustand der Nation spiegelte. Denn die gravierendsten Folgen der Sklaverei für die amerikanische Gesellschaft waren nach Auffassung Stowes die Zerstörung von Familien und Gemeinschaften als Orte, an dem moralische Werte gelebt und weitergegeben werden, und damit die moralische Verrohung des amerikanischen Volkes. Im Hinblick auf diese Botschaft leserlenkend wirken für die implizierte weiße Leserschaft unerwartete sprachliche Kreuzungen: Sklaven sprechen Standardenglisch, Sklavenhalter kreuzen ins „Neger-Englisch“. Dazu im Folgenden drei Beispiele.

Über Szenen in Sklavenunterkünften (Onkel Toms Hütte auf der ShelbyPlantage) und in von Sklaven dominierten Räumen (die Küche in Augustine St.Clares Haushalt) wird eine spezifische Black identity skizziert, die sich neben

35 MCDOWELl, 1931, S. 325; HOLTON, 1984, S. 70.

$36 \mathrm{Vgl}$. MeYer, 1994 und BURKETTE, 2001. Beide Autoren kommen durch den Abgleich der von Stowe verwendeten Merkmale mit soziolinguistischen Studien zu dem Ergebnis, dass Stowe realweltliche Charakteristika des Afroamerikanischen und verschiedener Südstaatenlekte verwendete. Die Nuancen in der Sprache der Charaktere führen sie auf Bildungsunterschiede und die natürliche Variabilität innerhalb von Sprachen wie auch Lekten zurück.

37 Vgl. FurNas, 1956, Rückeinband; Yarborough, 1986, S. 47; CANTAVE, 2007, S. 196.

38 Vgl. HOVET, 1989. 
traditionellen Gesängen und Speisen vor allem über das Black English als gemeinschaftsstiftender und -erhaltender Sprache konstruiert. Grundsätzlich nicht präsent sind in diesen Szenen Sklaven mit gemischtethnischer Vorfahrenschaft. Diese Mulatten- und Quadronenfiguren unterscheiden sich nicht nur durch ihre hellere Hautfarbe von den anderen Sklaven. Anders als die Sklaven dunklerer Hautfarbe weigern sie sich, die Unterjochung durch ihre weißen „Herren“ anzuerkennen. Unterstrichen wird die Verschiedenheit dieser Charaktere durch ihre standardenglische Sprache, in der etwa George Harris, kurz bevor er gen Norden flieht, seine Unzufriedenheit artikuliert: „,My Master! And who made him my master? That's what I think - what right has he to me? I'm as much a man as he is. [...W] What right has he to make a dray-horse of me? ${ }^{66^{39}}$ Harris verweist hier auf die von Stowe im gesamten Roman postulierte Gleichheit von Schwarz und Weiß auf der Ebene des Menschseins; eine Gleichheit, die ihm trotz seiner weißen Sprache und trotz seines weißen Vaters allein aufgrund seiner nur halbweiBen Hautfarbe unter der Sklaverei nicht zugestanden wird. Somit steht Harris exemplarisch für den „tragischen Mulatten“, der qua biologischer Herkunft zwei ethnische Identitätsgruppen vereint und doch von beiden ausgeschlossen bleibt; von der weißen aufgrund seiner Hautfarbe; von der schwarzen aufgrund seiner Verschiedenheit qua Sprache, Hautfarbe und Beziehung zu den weißen Sklavenhaltern. ${ }^{40}$ Schuld daran war, so Stowe, die Sklaverei. Die massenhafte Vergewaltigung schwarzer Frauen durch weiße Männer - nebst der Destruktivität dieser Praxis für weiße Familien - verursachte für gemischtethnische Sklaven eine Identitäts- und Zugehörigkeitsproblematik. Dazu wurde ihnen die Teilnahme an Gemeinschaft, die Stowe als Fundament der moralisch funktionierenden Gesellschaft ansah, verwehrt.

Mag das sprachliche Crossing von George Harris für Stowes weiße Leser noch mit ihrem Weltbild vereinbar gewesen sein, denn immerhin ist er halbweiß, dürften die Schwarz/Weiß-Kreuzungen in der Sprache des Protagonisten Uncle Tom und der seines letzten Masters Simon Legree für Irritation gesorgt haben. So spricht Tom als einziger der dunkelhäutigen Sklaven ein relativ merkmalsschwach ausgeprägtes Afroamerikanisch. Auf die Frage, weshalb er angesichts der grausamen Praktiken der Sklaverei nie den Glauben an Gott verloren hat, antwortet Uncle Tom:

39 STOWE, 2010, S. 14.

40 Die Außenseiterschaft gemischtethnischer Sklaven in der schwarzen Gemeinschaft beruhte weniger auf Selbstabgrenzung als auf der Tatsache, dass andere Sklaven ihnen aufgrund ihrer biologischen Nähe zu den weißen Sklavenhaltern häufig mit Misstrauen begegneten. Vgl. Blassingame, 1979, S. 211. 
[W] hen I was sold away from my old woman and children, I was jest a most broke up. I felt as if there warn't nothin' left; and then the good Lord, he stood by me, and he says, ,Fear not, Tom;' and he brings light and joy into a poor feller's soul, - makes all peace; and I's so happy, and loves everybody, and feels willin' jest to be the Lord's, and have the Lord's will done, and be put jest where the Lord wants to put me. ${ }^{41}$

Die wenigen Lektmerkmale in Toms direkte Rede sind vor allem phonetischer Natur, wie u.a. der Wegfall einzelner Laute und die Nasalisierung von ing-Endungen (nothin', willin'). Darüber hinaus finden sich Formen des sogenannten Augendialekts (jest, feller) ${ }^{42}$ sowie, charakteristisch für das Afroamerikanische, Doppelnegativkonstruktionen (warn't nothing) und die Nichtunterscheidung zwischen Singular und Plural bei Personalformen von Verben (I's, [I] loves). Als Einziger der Nicht-Mulatten bzw. Quadronenfiguren kreuzt Tom in das Standardenglisch der weißen Romanfiguren. Dadurch konstruiert sich auch sprachlich Toms Funktion als Mediator- und Vorbildfigur. Denn in seiner Jesusähnlich gelebten selbstlosen Nächstenliebe, selbst für die, die ihm Leid zufügen, ist Tom von seinem mundanen Minderheitenstatus entbunden und kann somit als Vorbild für Schwarz und Weiß gelten. Die moralische Katastrophe, die die Sklaverei für Stowe darstellte, konnte ihrer Ansicht nach nur durch eine Rückkehr Amerikas auf den moralisch tugendhaften Weg christlicher Nächstenliebe behoben werden.

Zum direkten Gegenspieler Toms und zur Verkörperung der von Stowe angeprangerten moralischen Verrohung der amerikanischen Gesellschaft wird in der zweiten Romanhälfte der brutale Plantagenbesitzer und Sklavenhalter Simon Legree. Gerne brüstet Legree sich mit der brutalen Geschäftsmäßigkeit, mit der er seine Sklaven als ersetzbare Waren behandelt:

Stout fellers last six or seven years; trashy ones gets worked up in two or three. I used to, when I fust begun, have considerable trouble fussin' with 'em [...] - doctorin' on 'em when they's sick, and givin' on 'em clothes and blankets, and what now, tryin' to keep 'em all sort o' decent and comfortable. Law, 't wasn't no sort o' use; [...] Now, you see, I just put 'em straight through, sick or well. When one nigger's dead, I buy another; and I find it comes cheaper and easier, every way. ${ }^{43}$

41 STOWE, 2010, S. 276.

42 Abweichungen von der standardmäßigen Schreibung, die in der Regel die Aussprache nicht verändern.

43 EBD., S. 310. 
Legrees rohe Ausdrucksweise weist wie die Sprache anderer weißer Sklavenhalter im Roman Kreuzungen sowohl in Varietäten der Südstaaten als auch ins Afroamerikanische auf. Diese unbewusste sprachliche Annäherung an seine Sklaven geht ironischerweise mit seinem Beharren auf der Primitivität und Subhumanität eben jener Sklaven einher. Noch deutlicher wird die auch sprachliche Entlarvung der weißen Sklavenhalter als die wahrhaft primitiven Glieder der amerikanischen Gesellschaft in einem Kommentar des Erzählers zum Gesprächsverhalten eines anderen Sklavenhalters: „His [Mr. Haley's] conversation was in free and easy defiance of Murray's Grammar. ${ }^{* 44}$

Aus den angeführten Bespielen lässt sich jedoch mitnichten schlussfolgern, alle weißen Figuren in Uncle Tom's Cabin seien per se Barbaren wie Legree, während alle schwarzen grundsätzlich Toms Güte zeigten. So zeigt die direkte Rede gutmütiger - aber freilich dennoch am Bestehen der institutionalisierten Sklaverei mitschuldhafter - Sklavenbesitzer Mr. Shelby und Augustine St.Clare im Gegensatz zu der Sprache von Legree keine lektalen Färbungen. Die Sprache von Sambo und Quimbo, die beiden brutalen schwarzen Aufseher auf der Plantage von Simon Legree, ist wiederum ob ihrer Merkmalsdichte schwer entzifferbar. Das brutale Duo repräsentiert eine Gruppe von Sklaven, die sich opportunistisch mit ihren weißen Herren und damit gegen ihre schwarzen Schicksalsgenossen verbünden. Ihre Sprache schlägt aber, als sie ihr Fehlverhalten einsehen und Tom vor dessen Tod reuig um Vergebung bitten, in beinahe lektfreies Standardenglisch um. Hierin wird einmal mehr der den gesamten Roman durchziehende Zusammenhang zwischen Sprache und Moral(losigkeit) deutlich.

Durch sprachliche Kreuzungen und die feine Nuancierung der Lektintensität der schwarzen und weißen Sprecher schuf Stowe auch sprachlich ein Mittel zur Identifizierung moralischer Bewertungsstandards im Roman. Mit wenigen Ausnahmen einer anderweitigen Funktionalisierung des Black English sprechen, unabhängig von der Hautfarbe, tendenziell moralisch schlechte Charaktere merkmalsdichteren Lekt als moralisch gute. ${ }^{45}$ Da höhere Merkmalsdichte in Uncle Tom's Cabin aufgrund der überwiegend lautmalerischen Darstellung der Lekte und der Abwandlung realweltlicher Lektspezifika auch mit erschwerter Lesbar-

44 EBD., S. 1.

45 Während das sehr merkmalsdichte Afroamerikanisch von Toms gutherziger Ehefrau, Tante Chloe, sowie der selbstbewussten Köchin Dinah in Augustine St.Clares Haus deren Funktion als Dreh- und Angelpunkt in der häuslich-familiären Gemeinschaft der Sklaven identifiziert, ist die stark lektifizierte Sprache des Sklavenmädchens Topsy Teil einer komisch-grotesken Darstellung, mit der Stowe stereotypen Vorstellungen von Schwarzen als von Natur aus wild und nicht zivilisierbar begegnete. 
keit einhergeht, entsteht beim Leser Empathie eher mit Charakteren, deren Sprache er mühelos verstehen kann, d.h. mit jenen schwarzen und weißen Figuren, die Stowe als moralisch vorbildhaft zeichnete.

Gleichzeitig postulierte Stowe mit ihrem Sprachgebungskonzept die Nichtexistenz einer typisch schwarzen und einer typisch weißen Sprache. Damit konterkarierte sie zum einen das Stereotyp des ob seiner inferioren Geisteskapazitäten grundsätzlich nur zu primitivem „Neger-Englisch“ fähigen Sklaven. Zum anderen unterstrich sie ihre Überzeugung von der menschlichen Gleichheit von Schwarz und Weiß mit deren sprachlicher Gleichheit.

\section{5. „UN DIGNE PENDANT DE ,UNCLE TOM'S CABIN"“: SPRACHKREUZUNGEN IN THE GRANDISSIMES}

Eine derart eindeutige lektale Markierung von moralisch guten und schlechten Charakteren lässt sich im Falle von George Washington Cables The Grandissimes kaum feststellen. Zwar spricht die mahnende moralische Stimme im Roman, der in den USA geborene Sohn deutscher Einwanderer Joseph Frowenfeld, merkmalsfreies Standardenglisch, aber dies gilt auch für Agricola Fusilier, den eigentlich frankophonen Patriarchen des Grandissimes-Clans und standhaften Vertreter des ancien régime. ${ }^{46}$ Dagegen ist das Englisch seines Neffen Honoré Grandissime, der sich im Verlauf des Romans immer deutlicher auf die Seite Frowenfelds schlägt, zumindest in der Erstveröffentlichung von Cables Text in der Zeitschrift Scribner's Monthly relativ stark dialektal eingefärbt, wenn auch nicht gar so intensiv wie bei Honorés anderen Verwandten, die an der Seite Agricolas stehen.

In anderer Hinsicht jedoch ist The Grandissimes dem 47 Jahre zuvor erschienenen Werk Stowes nicht unähnlich. Wie Uncle Tom's Cabin muss auch Cables Roman als politisches Werk gelesen werden, mit dem sich der Autor für die Rechte der Afroamerikaner in den Südstaaten der USA einsetzte (hier zur Zeit der Rekonstruktion, also der Epoche nach dem Bürgerkrieg und der offiziellen Abschaffung der Sklaverei). Anders als Stowe jedoch kleidet Cable seine politisch-moralische Botschaft nicht in eine religiöse, sondern eine historische Allegorie: The Grandissimes spielt im New Orleans des frühen 19. Jahrhunderts, genauer: in den Jahren 1803/1804, als sich die überwiegend französischstämmigen

46 Rosenwald argumentiert, Cable behalte das Standardenglische einem ,inneren Zirkel“ von Hauptfiguren vor, zumindest für die Zeitschriftenversion trifft dies jedoch nicht zu. Vgl. RoSENWALD, 2008, S. 73. 
Einwohner der Stadt, die sogenannten Kreolen, in der Folge des Louisiana Purchase mit einer als feindlich empfundenen Übernahme durch die US-amerikanische Administration und mit einer wachsenden Zahl von anglo-amerikanischen Einwanderern konfrontiert sahen und um ihre traditionelle Gesellschaftsform bangten. Bereits zeitgenössische Rezensenten erkannten die Analogie zur Situation New Orleans' bzw. der Südstaaten allgemein nach dem Ende des Bürgerkriegs und der Besatzung der ehemaligen konföderierten Staaten durch Militärgouverneure aus dem Norden. So schrieb etwa Hjalmar Hjorth Boyesen in seiner im November 1880 anonym erschienenen Rezension anlässlich der Buchveröffentlichung des Romans:

In fact, the state of affairs in Louisiana in 1804 is so nearly parallel with the state of affairs to-day, or at all events previous to 1876 , that to all intents and purposes the book is a study (and a very profound and striking one) of. Southern society during the period of reconstruction. Accordingly, we cannot help suspecting Mr. Cable of a benevolent intention to teach his countrymen some fundamental lessons of society and government, while ostensibly he is merely their dispassionate historian. ${ }^{47}$

Es waren jedoch nicht nur Cables im damaligen Kontext geradezu radikale politische Positionen - seine ebenfalls aus New Orleans stammende Schriftstellerkollegin Grace King sollte ihm später vorwerfen, er sei der Stadt in den Rücken gefallen $^{48}$-, sondern auch seine Darstellung der Kreolen und insbesondere von deren Sprache(n), die ihn, wie es sein späterer Illustrator Joseph Pennell formulierte, zum ,most cordially hated little man in New Orleans" machten. ${ }^{49}$

Wie in The Grandissimes, aber auch in anderen, nicht-fiktionalen Texten des Autors deutlich wird, sah Cable die Darstellung von Lekten in der Literatur als komplexes, sowohl ästhetisches als auch politisches Problem. Im Roman selbst äußert sich dies zum einen an mehreren Stellen, an denen sich der Erzähler über die Unzulänglichkeit des Mediums der gedruckten Sprache zur authentischen Wiedergabe von mündlicher Rede beklagt. So heißt es z.B. zu Beginn von Kapitel XXV:

Alas! the phonograph was invented three-quarters of a century too late. If type could entrap one-half the pretty oddities of Aurora's speech, - the arch, the pathetic, the grave, the earnest, the matter-of-fact, the ecstatic tones of her voice, - nay, could it but reproduce the

47 BOYESEN, 1880, S. 160. Vgl. EKSTRÖM, 1966, S. 155 Fn. 6.

48 KING, 1971, S. 60.

49 ROBINS, 1930, S. 56. 
movements of her hands, the eloquence of her eyes, or the shapings of her mouth, - ah! but type - even the phonograph - is such an inadequate thing! $!^{50}$

Hochman nimmt diese Stelle zum Anlass für seine Diskussion der zeitgenössischen Diskurse rund um Edisons Erfindung (s.o.), doch Cable erachtet selbst den Phonographen als unzureichend. Zum anderen wird in The Grandissimes Agricola Fusilier als Verfechter einer Sprachtheorie dargestellt, die streng nach Rassen und Ethnien hierarchisiert. ,, [S] he has picked up as many negro dialects as I know European languages"“" 51 sagt Agricola über eine Quadrone und unterscheidet damit grundlegend zwischen weißen und schwarzen Sprachformen, von denen Erstere das Etikett „Sprache“ verdienen, während Letztere lediglich als „Dialekte“ und damit als ,minderwertig ' bezeichnet werden. Doch auch innerhalb der Sprachen gelte es zu differenzieren: ,, English is not a language, sir; it is a jargon! [...] [Y] ou must not expect an old Creole to like anything in comparison with la belle langue. “52 Die generelle Darstellung Agricolas im Roman als hoffnungslos aus der Zeit gefallener, ,aged high-priest of a doomed civilization ${ }^{653}$ lässt Cables eigene Ansicht hierzu erahnen. In seinen nicht-fiktionalen Texten wandte sich Cable noch weitaus expliziter gegen rassistische Tendenzen in der Dialektliteratur; 1885 sprach er sich etwa in der Zeitschrift Critic vehement gegen die Praxis des Augendialekts aus: „Why is it that [writers such as Thomas Nelson Page] spell phonetically words which a Negro pronounces exactly the same as a white man does? ${ }^{654}$

Mögen Aussagen wie diese auch darauf hindeuten, dass Cable an einer möglichst authentischen und antirassistischen Darstellung von Dialekten gelegen war, so bekannte er jedoch 1897 in Current Literature: „It is probably always best that dialect should be sketched rather than photographed. "55 Brian Hochman vermutet, Cable habe hier eher die Notwendigkeit der Lesbarkeit von Dialektliteratur betonen als Kritik an möglichst authentischen Transkriptionen üben wollen. ${ }^{56}$ Doch vielleicht reflektiert Cables Aussage auch seine Erfahrungen mit der Rezeption von The Grandissimes. Zwar waren die zeitgenössischen Rezensionen zu Cables Werken überwiegend positiv ausgefallen, auch und gerade hinsichtlich

50 CABLE, 1879/1880, S. 698 [19, 5]. Vgl. auch EBD., S. 197 [20, 2].

51 EBD., S. $849[19,6]$.

52 EBD., S. $257[19,2]$.

53 EBD., S. $818[20,6]$.

54 Zit.n. HoCHMAN, 2010, S. 528.

55 Zit.n. EvANS, 1971, S. 217 f.

56 HOCHMAN, 2010 , S. 527. 
seiner Transkriptionen von Lekten. Der Atlantic Monthly z.B. schrieb über die Kurzgeschichtensammlung Old Creole Days (1879):

French and Spanish creoles, negroes, half-breed Indians, and Américains of every grade circulate gayly through his pages, meet and part with immense evolution of electricity; and "we hear them speak each in his own tongue, "for the author's mastery over mongrel dialects is something marvelous. Surely never before were such novel and varied vocal effects represented by the twenty-six letters of the English alphabet and a few italics and apostrophes. $^{57}$

Kritisiert wurde allenfalls die mangelnde Lesbarkeit von Cables Dialekten, wie z.B. in Harper's Magazine: ,The dialect dialogues with which the story is profusely garnished have often little to commend them beyond their singularity, and although sufficiently novel and curious to amuse the reader at first, finally become tedious by their excess. “58

Ungleich heftiger fielen jedoch die Reaktionen $\mathrm{zu}$ The Grandissimes aus New Orleans aus, insbesondere seitens der Kreolen. Neben zahlreichen kritischen Artikeln in der frankophonen Zeitung L'Abeille de la Nouvelle-Orléans stechen insbesondere zwei Publikationen heraus. ${ }^{59} \mathrm{Im}$ Jahr 1880 veröffentlichte der Abbé Adrien Roquette die Schmähschrift Critical Dialogue between Aboo and Caboo on a New Book or a Grandissime Ascension, in der er Agricola Fusilier unter dem Namen „Aboo" wiederaufleben lässt und Cables Roman als ,an unnatural, Southern growth, a bastard sprout, un digne pendant de ,Uncle Tom's Cabin" " bezeichnet. ${ }^{60}$ Aboos Dialogpartner Caboo fällt ein vernichtendes Urteil über Cables Lekte:

Had we but the hissing, whistling, howling drunken ,mob“ of tortured English words, it might be tolerable; but we have also the worst patois that ever grated the human ear, in savage discord of sounds. Never before were verbs, nouns and adjectives so multitudinously disallied, tied together, whipped into forced union. ${ }^{61}$

57 N.N., 1880a, S. 44.

58 N.N., 1880b, S. 153.

59 Vgl, TINKER, 1934, S. 324.

60 ROQUETTE, 1880, S. 18.

61 EBD., S. 12. 
Fünf Jahre später brachte der bekannte kreolische Historiker Charles Gayarré die kreolische Kritik an Cables Dialektliteratur auf den Punkt. In The Creoles of History and the Creoles of Romance schreibt er:

If Mr. Cable has represented the most distinguished of our creole families as having forgotten to speak French, and as using only the jargon which the negroes had constructed out of that language, this invention would have far exceeded the limits of those liberties which fancy in its wildest flights may be permitted to take with common sense. But when he makes them prefer, not the French, not the creole negro patois, but the broken English of the negroes of Virginia, the Carolinas, Georgia, etc. the perversion of his intellect becomes overpowering and incomprehensible. ${ }^{62}$

Stein des Anstoßes ist für Gayarré somit die Tatsache, dass die im Roman von den kreolischen Charakteren verwendeten Varianten des Englischen und Französischen von afroamerikanischen Einflüssen zeugten - einerseits das z.T. sehr merkmalsdichte Englisch, das William Evans in einer Studie treffend als „French-English literary dialect“ bezeichnet hat, ${ }^{63}$ andererseits das selbst für Frankophone beinahe gänzlich unverständliche ,patois“. Hatten einige Kritiker die Verwendung dieser Sprachvarianten vor allem als dem sozialen Stand der Charaktere unangemessen erachtet, ${ }^{64}$ stand für Gayarré der Aspekt der Rasse im Vordergrund. Cable, so der generelle Vorwurf Gayarrés, stelle die Kreolen als linguistisch, kulturell und letztlich auch bezüglich ihrer Abstammung hybrides Volk dar.

Damit lag Gayarré nicht gänzlich falsch. In Kapitel IV des Romans schreibt Cable etwa zum Stammbaum der Familie Grandissime, die stellvertretend für alle Kreolen steht: „But the true, main Grandissime stock [...] has kept itself lilywhite ever since France has loved lilies - as to marriage, that is; as to less responsible entanglements, why, of course. ${ }^{65}$ Zahlreiche kreolische Charaktere, darunter insbesondere Aurora de Grapion, übernehmen kulturelle Praktiken der Sklaven wie z.B. den Voodoo-Kult. Und das Black English der Sklaven und freien Quadronen stellt, wie Evans bemerkt, zumindest eine der Quellen für den „French-English literary dialect“ der Kreolen im Roman dar. ${ }^{66}$

62 GAYARRE, 1885 , S. $18 \mathrm{f}$.

63 EVANS, 1971.

64 Vgl. EBD., S. $216 f$.

65 CABLE, 1879/1880, S. $106[19,1]$.

66 EVANS, 1971, S. 215. 
Im offen rassistischen Kontext der Südstaaten des späten 19. Jahrhunderts kam eine solche Darstellung der Kreolen - ob historisch fundiert oder nicht - jedoch einem Affront gleich. Mit der Abschaffung der Sklaverei nach dem Bürgerkrieg und insbesondere mit dem Wegfall der Unterscheidung zwischen Sklaven und freien Afroamerikanern hatten sich die sozialen Fronten in Louisiana neu zu formieren begonnen. Dazu wurde die alte Rivalität zwischen FrankoAmerikanern (Kreolen jedweder Abstammung) und Anglo-Amerikanern zunehmend durch den rein auf Rasse basierenden Konflikt zwischen Schwarz und Weiß ersetzt. Um ihren ohnehin verminderten ökonomischen, politischen und sozialen Stand nicht noch weiter zu gefährden, insistierten die Kreolen auf eine rein europäische Identität: „By the end of the nineteenth century Creole and American judges and lawmakers alike were vigorously policing the racial boundaries of Louisiana with a one-drop rigor unimaginable at the time of the Louisiana Purchase. “67 Cables Roman, noch dazu ein nationaler Bestseller, schien ohnehin schon virulente Gerüchte, nach denen es sich bei den Kreolen eben nicht um rein weiße Abkömmlinge französischer Siedler handelte, nur noch weiter $\mathrm{zu}$ befeuern und wurde gerade deshalb von den Kreolen so heftig attackiert. $^{68}$

Interessanterweise wurde Cable häufig als Südstaatler charakterisiert, der lediglich aus Gier nach Ruhm und Geld den Nordstaaten nach dem Munde schrieb, so etwa von der bereits erwähnten Grace King (s.o.). Gayarré dagegen warf Cable vor, „one accidentally born“ in Louisiana zu sein und „claiming by virtue of that accident the right, not only to speak in the name of Louisiana, but also of the whole South ". ${ }^{69}$ Für Gayarré war Cable ein Außenseiter, der böswillig und ohne jegliche Legitimation über die Geschichte, den Charakter und die Bräuche der Kreolen schrieb und sich deren Sprache aneignete; er war, kurzum, ein Kreuzer. Cables Rezeption durch Gayarré und andere Kreolen bestätigt letztlich, was verschiedene Forscher über Crossing allgemein sagen: Die Verwendung eines fremden Codes kann, so schreibt etwa Androutsopoulos, „Einschränkungen unterliegen, da sie situationsspezifisch als diskriminierend empfunden [...] werden kann" “ ${ }^{70}$ Die Kreolen, und allen voran Gayarré, sahen sich durch The Grandissimes diskriminiert und kämpften daher gegen Cables Crossing an.

67 Powell, 2008, S. 21. Vgl. DoMingueZ, 1986, S. 134-147.

$68 \mathrm{Vgl}$. HANDLEY, 2000, S. 63.

69 GAYARRE, 1885, S. $18 \mathrm{f}$.

70 ANDROUTSOPOULOS, 2002, S. 31. 


\section{Von KREUzUng zU KREUZUng: DialektLiteratur ALS MEDIALE QUELLEN FÜR CROSSINGS}

Uncle Tom's Cabin und The Grandissimes stellen nun nicht nur selbst Fälle von sprachlichen bzw. literarischen Kreuzungen dar. Als zu ihrer Zeit überaus erfolgreiche Romane fungierten sie auch als Quellen für weitere, z.T. „klassische“ und literarische Crossings. ${ }^{71}$ So wird etwa von Mark Twain und William Dean Howells berichtet, sie hätten sich bei einem Treffen nach der gemeinsamen (lauten) Lektüre von The Grandissimes den Rest des Tages auf "Creole“ unterhalten. Howells selbst schrieb Cable, er spreche nur noch „Creole" mit seiner Frau. ${ }^{73} \mathrm{Im}$ Falle von Uncle Tom's Cabin kam es sogar zu weiteren literarischen - oder besser: medialen - Kreuzungen. In den zahlreichen deutschsprachigen Übersetzungen von Stowes Klassiker werden zwar bis heute im Stile des eingangs zitierten Dr. Ungewitter sämtliche den englischen Text auszeichnenden sprachlichen Kreuzungen durch Wiedergabe der Sprache der Dialoge im Standarddeutschen praktisch zurïckgenommen. Dadurch ist in den deutschen Übersetzungen nicht nur die Unterscheidung zwischen Schwarz und Weiß zumindest sprachlicherseits aufgehoben, sondern auch die beschriebenen Effekte der von Stowe vorgenommenen sprachlichen Nuancierung auf der Grundlage moralischer Prinzipien und der abolitionistischen Botschaft des Romans.

Weitaus interessanter im Hinblick auf language crossings ist dagegen die bislang einzige Spielfilmadaption des Romans; ${ }^{74}$ eine deutsch-italienische Produktion, die 1965 in deutscher Sprache unter dem Titel Onkel Toms Hütte Premiere feierte. ${ }^{75}$ Wie in der englischsprachigen literarischen Vorlage sprechen die

71 Medientexte gelten neben persönlichen Kontakten als Hauptressource für sprachliche Kreuzungen; Androutsopoulos spricht etwa von einer „Gleichzeitigkeit direkter und medialer Ressourcen“ (2002, S. 20). Das Besondere an den vorliegenden Fällen ist, dass es sich bereits bei den Medientexten selbst um Kreuzungen handelt.

72 Vgl. TURNER, 1966, S. 122.

73 Vgl. RosenwaLd, 2008, S. 9.

74 Obschon Uncle Tom's Cabin mit mindestens neun bekannten Produktionen zwischen 1903 und 1927 zu den am meisten verfilmten literarischen Stoffen der Stummfilmära gehörte, wurde der Roman in den Vereinigten Staaten seither nie wieder filmisch adaptiert. Nur einmal, 1946, gab es konkrete Pläne für eine Verfilmung, die aber nach massiven Protesten der National Association for the Advancement of Colored People fallen gelassen wurden. 1987 wurde eine von der britischen BBC produzierte TVFassung ausgestrahlt, die jedoch nie ins Deutsche synchronisiert wurde. 
Charaktere unterschiedliche Lekte bzw. Akzente, die ihnen jedoch anders als bei Stowe eindeutig aufgrund ihrer Hautfarbe zugeordnet werden. So sprechen die Sklaven um Onkel Tom ein mehr oder weniger stark amerikanisch akzentuiertes Deutsch. Dieses stellt keinen ethnischen Bezug her, da Deutsch-Muttersprachler, würde man die Sprecher nicht sehen, assoziieren würden, es handele sich um einen qua Hautfarbe beliebigen Deutsch sprechenden Amerikaner. Vielmehr lässt der Film die schwarzen Figuren in eine „Lernersprache ${ }^{476}$ kreuzen, die nämlich des Deutsch lernenden Amerikaners. Im Zusammenspiel mit den Szenen, in denen Lese- und Schreibdefizite schwarzer Charaktere exponiert werden - Onkel Tom liest die Bibel so stockend wie ein Grundschüler - wird dadurch mehr als die in der Tat bildungsmäßige Benachteiligung der Sklaven auf den Baumwollplantagen in den Südstaaten des 19. Jahrhunderts konnotiert.

Demnach erscheint die Sprache der schwarzen Figuren neben dem prävalierenden Deutsch der weißen Südstaatler exotisch; ein Eindruck, der verstärkt wird durch die Tatsache, dass der Film das Schicksal der Sklaven ohnehin nur marginal behandelt. Im Mittelpunkt stehen - ganz im Stile der deutsch-österreichischen Heimatfilme à la Sissi der 1950er Jahre - heimelige Familien- und kitschige Liebesszenen in den feudalen Anwesen der weißen Sklavenhalter, die ein verklärtes Bild der Antebellumzeit zeichnen. Nur folgerichtig erscheint vor diesem inhaltlichen Hintergrund die sprachliche Kreuzung, die der Film auf Seiten der weißen Sprecher vornimmt: O.W. Fischer leiht dem im Roman Standardenglisch sprechenden, patriotischen Südstaatler Augustine St.Clare im Film seinen österreichischen Akzent. Dadurch entsteht, wie Der Spiegel kurz nach der Erstaufführung des Films urteilte, „die Illusion, der Mississippi fließe im Donautal ", ${ }^{77}$ und damit über die sprachliche Kreuzung hinaus eine geographische und kulturelle Crossing-Situation.

\section{FAZIT}

Zu Beginn des 20. Jahrhunderts geriet die Dialektliteratur in den USA zusehends aus der Mode. Mit dem makrohistorischen Schwellenkontext, den zahlreichen sozialen, politischen und wirtschaftlichen Umbrüchen des Bürgerkriegs, der Reconstruction und des Gilded Age, schien auch der Einsatz von Lekten, wie sie von Autoren wie Cable und Stowe verwendet wurden, als ästhetische Strategie

76 Vgl:HUNEKE/STEINIG, 2013, S. 40-48.

77 N.N., 1965. 
zur literarischen Verhandlung der sich durch diese Umbrüche ergebende Neuverteilung von sozialen Rollen zu verschwinden.

Die Verwendung in diesem liminalen Kontext stellt jedoch nur eine der zahlreichen Parallelen zwischen „klassischen“ und literarischen Kreuzungen dar. So können die Romane von George Washington Cable und Harriet Beecher Stowe exemplarisch als die Pole jener für die amerikanische Dialektliteratur des 19. Jahrhunderts charakteristischen multifunktionalen Verwendung von regional, sozial und ethnisch markierten Lekten gelten: Während sich in beiden Romanen Kreuzungen mit politischen Botschaften verbinden, war Cable in The Grandissimes darum bemüht, Lekte möglichst exakt zu verschriftlichen. Damit wandte er sich insbesondere gegen die selektive Praxis des Augendialekts. Stowe dagegen setzte in Uncle Tom's Cabin von ihren Vorbildern stark abgewandelte lektale Merkmale gezielt zur Leserlenkung ein. Dadurch sind die hier vorgestellten literarischen Kreuzungen hinsichtlich ihrer über den Sprech- bzw. Schreibakt hinausgehenden Konnotationen dem „klassischen“ Crossing nicht unähnlich.

Wie die Rezeption beider Werke schließlich ebenfalls gezeigt hat, stellen literarische Kreuzungen, ähnlich wie „klassische“ Kreuzungen, aber immer auch sprachliche Minenfelder dar. Stowe löste mit ihrem Roman - so wird jedenfalls Abraham Lincoln in einer berühmten Anekdote zitiert - den Bürgerkrieg aus. ${ }^{78}$ Cable sorgte mit seinem Buch (sowie seinen später publizierten politischen Essays) in seiner Heimatstadt und in den Südstaaten generell für solche Kontroversen, dass er es 1885 vorzog, nach Northampton in Massachusetts zu ziehen. Wer in welchem Kontext welche (fremde) Sprache verwendet, ist folglich auch und gerade in der Literatur von Belang.

\section{LITERATUR}

ANDROUTSOPOULOS, JANNIS: jetzt speak something about italiano. Sprachliche Kreuzungen im Alltagsleben, in: Osnabrücker Beiträge zur Sprachtheorie 65 (2002), S. 5-35.

Blassingame, JoHn W.: The Slave Community. Plantation Life in the Antebellum South, New York/Oxford 1979 (1940).

BOYESEN, HJALMAR H.: Cable's „Grandissimes“, in: Scribner's Monthly 21, 1 (1880), S. 159-161.

BuRketTe, Allison: The Use of Literary Dialect in Uncle Tom's Cabin, in: Language and Literature 10, 2 (2001), S. 158-170. 
Cable, George W.: The Grandissimes. A Story of Creole Life, in: Scribner's Monthly 19, 1-20, 2 (November 1879-August 1880).

CANTAVE, SOPHIA: Who Gets to Create the Lasting Images? The Problem of

Black Representation in Uncle Tom's Cabin, in: Harriet Beecher Stowe's Uncle Tom's Cabin. A Casebook, hg. von ELIZABETH AMMONS, Oxford 2007, S. 193-205.

DOMINGUEZ, VIRgINIA: White by Definition. Social Classification of Creole Louisiana, New Brunswick 1986.

EkSTROM, KJELL: George Washington Cable. A Study of His Life and Work, New York 1966.

Evans, WILliam: French-English Literary Dialect in The Grandissimes, in: American Speech 46, 3-4 (1971), S. 210-222.

FloREY, KenNETH: Stowes's Uncle Tom's Cabin, Explicator 45, 1 (Herbst 1986), S. 20-21.

FREITAG, FLORIAN: Rencontres américaines. English-French Relations in Kate Chopin's Short Stories and the Cultural Work of Local Color Fiction, in: Amerikastudien/American Studies 58, 3 (2013), S. 409-426.

FREUNEK, SIGRID: Literarische Mündlichkeit und Übersetzung. Am Beispiel deutscher und russischer Erzähltexte, Berlin 2007.

FURNAS, JOSEPH C.: Goodbye to Uncle Tom, New York 1956.

GARLAND, HAMLIN: Main-Travelled Roads, New York 1918.

GAYARRE, CHARLES: The Creoles of History and the Creoles of Romance, New Orleans 1885.

HANDLEy, GEORGE B.: Postslavery Literatures in the Americas. Family Portraits in Black and White, Charlottesville 2000.

HARDWIG, BILL: Upon Provincialism. Southern Literature and National Periodical Culture, 1870-1900, Charlottesville 2013.

HARRold, Stanley: Gamaliel Bailey, Antislavery Journalist and Lobbyist, in: In the Shadow of Freedom. The Politics of Slavery in the National Capital, hg. von PAUL FINKELMAN/DONALD R. KENNON, Athens 2011, S. 58-82.

HINNENKAMP, VOLKER: Mehrsprachigkeit in Deutschland und deutsche Mehrsprachigkeit. Szenarien einer migrationsbedingten Nischenkultur der Mehrsprachigkeit, in: Das 20. Jahrhundert. Sprachgeschichte - Zeitgeschichte, hg. von HEIDRUN KäMPER/HARTMUT SCHMIDT, Berlin 1998, S. 137-162.

HOCHMAN, BRIAN: Hearing Lost, Hearing Found. George Washington Cable and the Phono-Ethnographic Ear, in: American Literature 82, 3 (September 2010), S. 519-551.

Holton, Sylvia W.: Down Home and Uptown. The Representation of Black Speech in American Fiction, London/Toronto 1984. 
Hovet, THeOdore R.: The Master Narrative. Harriet Beecher Stowe's Subversive Story of Master and Slave in Uncle Tom's Cabin and Dred, Lanham/London 1989.

HUNEKe, HANS-WeRnER/STEINIG, WolfGANG: Deutsch als Fremdsprache. Eine Einführung, Berlin 2013.

JONES, Gavin: Strange Talk. The Politics of Dialect Literature in Gilded Age America, Berkeley 1999.

KING, GRACE: Memories of a Southern Woman of Letters, Freeport 1971.

KirKham, Edwin B.: The Building of Uncle Tom's Cabin, Knoxville 1977.

McDowell, TRemaine: The Use of Negro Dialect by Harriet Beecher Stowe, American Speech 6, 5 (Juni 1931), S. 322-326.

MEYER, MICHAEL: Toward a Rhetoric of Equality. Reflective and Refractive Images in Stowe's Language, in: The Stowe Debate, hg. von M.I. LowancE/ E.E. WeTSBROOK/R.C. ProsPo, Amherst 1994, S. 236-254.

N.N.: Old Creole Days and Other Novels, in: The Atlantic Monthly 45, 267 (Januar 1880), S. 44-53. [1880a]

N.N.: Editor's Literary Record, in: Harper's New Monthly Magazine 62, 367 (Dezember 1880), S. 149-155. [1880b]

N.N.: Onkel Toms Hütte, in: Der Spiegel 19 (1965), http://www.spiegel.de/ spiegel/print/d-46272536.html (aufgerufen: 19.11.2015).

Onkel Toms Hütte (BRD/I 1965, R: GÉZA vON RADVÁNYI).

Parfait, Claire: The Publishing History of Uncle Tom's Cabin, 1852-2002, Burlington 2007.

PAUL, Heike: Kulturkontakt und Racial Presences. Afro-Amerikaner und die deutsche Amerika-Literatur, 1815-1914, Heidelberg 2005.

Powell, LAwRENCE N.: Introduction. A Novelist Turns Historian, in: The New Orleans of George Washington Cable. The 1887 Census Office Report, hg. von DEMS., Baton Rouge 2008, S. 1-35.

RAMPTON, BEN: Crossing. Language and Ethnicity among Adolescents, London/New York 1995.

DERS.: Language Crossing and the Redefinition of Reality, in: Code-Switching in Conversation. Language, Interaction and Identity, hg. von PETER AUER, London/New York 1998, S. 290-321.

RoBins, ElizaBeth: The Life and Letters of Joseph Pennell. With Illustrations, Boston 1930.

ROQUETTE, ADRIEN: Critical Dialogue between Aboo and Caboo on a New Book or a Grandissime Ascension, Mingo City [New Orleans] 1880.

ROSENWALD, LAWRENCE A.: Multilingual America. Language and the Making of American Literature, Cambridge 2008. 
STOWE, HARRIET B.: Onkel Tom's Hütte oder Negerleben in den Sclavenstaaten des freien Nordamerika. Übersetzt und bearbeitet von DR. UNGEWITTER, Wien/Lepizig 1852.

DIES.: Uncle Tom's Cabin, New York/London 2010 (1852).

TINKER, EDWARD L.: Cable and the Creoles, in: American Literature 5 (1934), S. 313-326.

TURNER, ARLIN: George W. Cable. A Biography, Baton Rouge 1966.

Vollaro, Daniel R.: Lincoln, Stowe, and the „Little Woman/Great War" Story. The Making, and Breaking, of a Great American Anecdote, in: Journal of the Abraham Lincoln Association, 30, 1 (2009), S. 18-34.

YARBOROUGH, RICHARD: Strategies of Black Characterization in Uncle Tom's Cabin and the Early Afro-American Novel, in: New Essays on Uncle Tom's Cabin, hg. von ERIC J. SUNDQUIST, Cambridge 1986, S. 45-84. 


\section{DuEPublico}

Duisburg-Essen Publications online

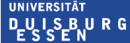

offen im Denken

Ub $\mid \begin{gathered}\text { universitäts } \\ \text { bibliothek }\end{gathered}$

Dieser Text wird über DuEPublico, dem Dokumenten- und Publikationsserver der Universität Duisburg-Essen, zur Verfügung gestellt. Die hier veröffentlichte Version der E-Publikation kann von einer eventuell ebenfalls veröffentlichten Verlagsversion abweichen.

DOI: $\quad 10.14361 / 9783839435380-004$

URN: urn:nbn:de:hbz:464-20201201-161824-5

Freitag, Florian; Rosbach, Annika: Literarische Crossings : Sprachliche Kreuzungen in der amerikanischen Dialektliteratur des 19. Jahrhunderts. In: Crossing : Über Inszenierungen kultureller Differenzen und Identitäten / Dresen, Antje ; Freitag, Florian (Hrsg.). Bielefeld: Transcript, 2017, S. 89 - 116. eISBN: 978-3-8394-3538-0 - DOI: https://doi.org/10.14361/9783839435380

(C) 2017 transcript Verlag Alle Rechte vorbehalten. 